\title{
Erratum to: Ideal Gas Outflow from a Cylindrical or Spherical Source into a Vacuum
}

Kh. F. Valiev ${ }^{a}$ and A. N. Kraiko ${ }^{a, b, *}$

${ }^{a}$ Baranov Central Institute of Aviation Motors (CIAM), ul. Aviamotornaya 2, Moscow, 111116 Russia

${ }^{b}$ Moscow Institute of Sciences ana Technology, Institutskii per. 9, Dolgoprudnyi, Moscow oblast, 141701 Russia

*e-mail: akraiko@ciam.ru

Received July 12, 2019

DOI: $10.1134 / \mathrm{S} 0015462819050136$

On page 599 in formulas (1.3) instead of

should read

$$
x=\left[\frac{\gamma-1}{a^{2}\left(\gamma+1-2 a^{2}\right)}\right]^{1 /[2(v-1)]}
$$

$$
x=\left[\frac{\gamma-1}{a^{4 /(\gamma-1)}\left(\gamma+1-2 a^{2}\right)}\right]^{1 /[2(v-1)]},
$$

and in equations (2.1) instead of

should read

$$
\frac{d t}{d a}=2 \frac{(\gamma-1)^{(1-v / 2) /(v-1)}\left[\left(\gamma+1-2 a^{2}\right)^{1 / 2}+(\gamma-1)^{1 / 2} a\right]}{(v-1) a^{1+2 /[(v-1)(\gamma-1)]}\left(\gamma+1-2 a^{2}\right)^{1+1 /[2(v-1)]}}
$$

$$
\frac{d t}{d a}=\frac{2(\gamma+1)(\gamma-1)^{(1-v / 2) /(v-1)}\left(a^{2}-1\right)}{(v-1) a^{1+2 /[(v-1)(\gamma-1)]}\left(\gamma+1-2 a^{2}\right)^{1+1 /[2(v-1)]}\left[\left(\gamma+1-2 a^{2}\right)^{1 / 2}-(\gamma-1)^{1 / 2} a\right]} .
$$

All results of the paper are obtained using the correct formula and equation.

The original article can be found on-line at https://doi.org/10.1134/S0015462818050154 